\title{
Using Modified Couple Stress Theory to Investigate the Size-Dependent Instability of Rotational Nano-Actuator under Van der Waals Force
}

\author{
${ }^{* 1}$ Yaghoub Tadi Beni \\ ${ }^{* 1}$ Faculty of Engineering, Shahrekord University, Shahrekord, Iran
}

\begin{abstract}
While experiments reveal that the mechanical response of nano-structures is size-dependent, classic continuum theories are not able to simulate this size effect. In this paper, a non-classic continuum theory e.g. modified couple stress theory is applied for modeling the size dependent instability of torsional nano-actuator. The constitutive equation of the actuator is derived taking the effect of electrostatic and molecular forces into account. Variation of the tilt angle as a function of the applied voltage is obtained and the instability parameters i.e. instability voltage and instability angle are determined. Two actuators with different cross-sectional torsional beams are investigated as case studies. Results show that when the thickness of the torsional beam is comparable with the intrinsic material length scale, size-dependency of material characteristics can highly affect the instability parameters of the actuator. The effect of van der Waals ( $\mathrm{vdW}$ ) molecular forces on the size-dependent instability is investigated. Furthermore, the minimum gap between the mirror and the ground to ensure that the actuator does not adhere the substrate (due to molecular force) is computed. It is found that proposed model is able to predict the experimental results more accurately than the previous classic models.
\end{abstract}

Keywords: Rotational nano-actuator, Size effect, Modified couple stress theory, Pull-in instability, van der Waals force

\section{Introduction}

With recent advanced in nanotechnology, torsional actuators become one of the most important elements in developing nano-electromechanical systems (NEMS) and micro-opto-electromechanical systems (MOEMS) [1,2]. The rotational nano-actuators have wide range of applications i.e. microscopic measurements, laser telecommunication, developing micr-robots, etc.. Consider a typical torsional nanoactuator in Fig.1 which is constructed from movable conductive component (mainplate) which is suspended over a rigid fixed conductive component (ground substrate). This type of ultra-small structure has attracted attentions of many experimental and theoretical researchers [3-5]. Applying a voltage difference between movable and fixed components rotates the movable component. By increasing the voltage, the electrostatic torque overcomes the mechanical torsion resistance. Hence, the mainplate tilts abruptly into the substrate and the actuator becomes unstable. This phenomenon that is known as pull-in instability is a crucial issue in design actuators. Determining the pull-in instability parameters, i.e. pull-in voltage and angle, is crucial in torsional actuator design. In recent decades, simulation of the torsional actuators at micro-scales (without considering nano-scale effects) has been conducted by many researchers [6-10]. Previous investigators have applied one-degree-of-freedom (1DOF) models to calculate the pull-in parameters of torsional actuator [9-12]. Since the vertical displacement of the movable component is usually small, these models provide reliable results in most cases. Other researchers have used a more precise 2DOF model to capture the torsion/bending coupled pull-in instability of micro-actuators [13-19].

With a decrease in dimension from micron to nanometer, the presence of van der Waals (vdW) force can highly affect the electromechanical behavior of the nanostructures. When the separation between the conductive electrodes is typically less than several nanometers, the $\mathrm{vdW}$ force can considerably influence the stable performance of the system. Effect of vdW force on performance of nanoswitches [20], nano-plates [21], nanotube-based actuators [22] and nano-bridges [23] has been

*Corresponding author: Faculty of Engineering, Shahrekord University, Shahrekord, Iran, E-mail: tadi@eng.sku.ac.ir, Phone: +983814424438 Fax: +983814424438 
previously investigated. Other researchers have studied the pull-in behavior of rotational systems considering the effect of vdW attraction [17, 24-28].

Apart from the vdW attraction, another nano-scale phenomenon that can highly affect the electromechanical behavior of systems is size dependency of material characteristics. When characteristic size (thickness, diameter, etc.) of a micro/nano element is in the order of its intrinsic material length scales (typically sub-micron), the material elastic constants depend on the element dimensions. It is well-established that mechanical behaviors of micro/nano structure are size dependent $[29,30]$. Fleck et al. has reported the size dependency of material for torsional elements [29]. In this regard, size effect is a very important phenomenon that should be considered in modeling the rotational actuators with micro/nano torsional beams. Note that classical continuum mechanics is unable to simulate the size effect in micro/nano structures. Therefore, non-classic continuum theories such as nonlocal [31], strain gradient [30] and modified couple stress [32] theories have been developed to model the size dependent behavior of ultra-small structures. These theories utilize additional material length scale parameters and are able to capture the sizedependency of the constitutive material. Recently, new modified couple stress theory has been proposed by Yang et al. [32]. According to their theory, two material constants in couple stress theory are reduced to only one length scale parameter. In this view, this modified theory has been applied to model microbeams by many researchers [33-37].

Modified couple stress theory has been used to investigate the size-dependent pull-in instability of beam-type NEMS [38]. However, to the best knowledge of the authors, usage of modified couple stress theories for modeling the size-dependent instability of rotational actuators have not been addressed in the literature.
Herein, the modified couple stress theory has been applied to model the effect of size dependency of material characteristics on pull-in instability of rotational nano-actuators. The governing equation of the system has been developed considering the presence of $\mathrm{vdW}$ force. The pull-in parameters are determined and effect of size dependency of material characteristics on the pull-in parameter is discussed.

\section{Governing Equation}

Figure 1 shows schematic views of electrostatic torsional actuator. The torsional mainplate suspended by two elastic torsional nano-beams over the substrate electrodes. In this model, small deformation assumptions is used which acceptable in the MEMS/NEMS literature. In this section the governing equation of the rotational actuator is derived. The list of symbols used in this article is presented in Table 1.

Table 1. Summary of all design variables for the torsional mirror

\begin{tabular}{|c|c|}
\hline$E$ & $\begin{array}{l}\text { Young's modulus of the torsion nano- } \\
\text { beam }\end{array}$ \\
\hline$\square$ & Poisson ratio of the torsion nano-beam \\
\hline$\mu=E / 2(1+\square)$ & Shear modulus of the torsion beam \\
\hline$a$ & Length of the main plate \\
\hline$b$ & Width of the main plate \\
\hline$L$ & Length of the torsion nano-beams \\
\hline$D$ & Gap between main plate and electrode \\
\hline$a_{1}$ & Inner distance between two electrodes \\
\hline$a_{2}$ & Outer distance between two electrodes \\
\hline$V$ & $\begin{array}{l}\text { Applied voltage between the main plate } \\
\text { and one electrozde }\end{array}$ \\
\hline $\begin{array}{l}\square=8.854 \times 10^{-} \\
\bar{A}=(04-4) \times 10^{-19} \mathrm{~J}\end{array}$ & $\begin{array}{l}\text { Permittivity of vacuum } \\
\text { Planck's constant divided by } 2 \pi\end{array}$ \\
\hline & Pull-in voltage \\
\hline$\Theta_{P I}$ & Normalized pull-in angle \\
\hline
\end{tabular}

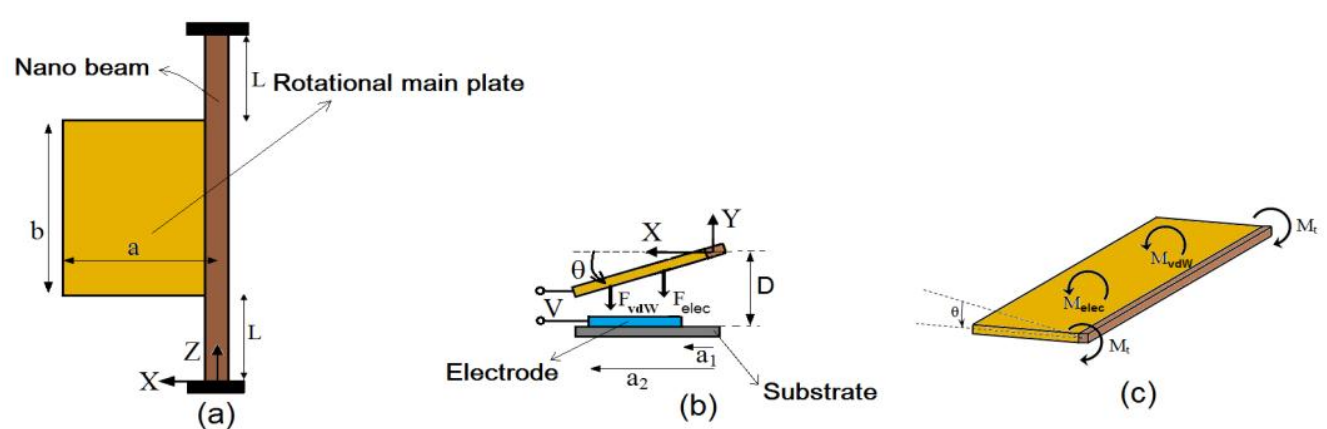

Figure 1. Schematics view of torsional nano-mirror 
The minimum total energy principal is used to obtain the equilibrium governing equation of the nanoactuator under the $\mathrm{vdW}$ and electrical forces. According to this principal, at the equilibrium state, the variation of the total potential energy of the system with respect to all degrees of freedom is zero. That the total potential energy of the torsional mirror can be divided into the potential strain energy (torsion nano-beams) and the energy of external loads (electrostatic and vdW forces). Considering the rigid mainplate in Figure 1, the total potential energy is determined as

$$
\Pi=2 \mathrm{~W}_{\text {elas }}+\mathrm{W}_{\text {elec }}+\mathrm{W}_{\mathrm{vdW}}
$$

where $\Pi$ is the total potential energy of the system, $\mathrm{W}_{\text {elas }}, \mathrm{W}_{\text {elec }}$ and $\mathrm{W}_{\mathrm{vdW}}$ are the work of elastic moment, electrostatic and $\mathrm{vdW}$ forces, respectively. In equilibrium point, the variation of the total potential energy of the system is zero. Therefore we have:

$$
\delta \Pi=2 \delta \mathrm{W}_{\text {elas }}+\delta \mathrm{W}_{\text {elec }}+\delta \mathrm{W}_{\mathrm{vdW}}=0
$$

In the following the variation of $\mathrm{W}_{\text {elas }}, \mathrm{W}_{\text {elec }}$ and $\mathrm{W}_{\mathrm{vdW}}$ are calculated.

$$
\delta \mathrm{W}_{\text {elas }}=\frac{\mu \theta}{\mathrm{L}}\left(\mathrm{J}+\mathrm{J}_{\mathrm{c}}\right) \delta \theta
$$

It should be noted that $\mathrm{J}_{\mathrm{c}}$ is a function of $l$ which is the length scale parameter applied in the modified couple stress theory [28].

$\delta \mathrm{W}_{\text {elec }}=\frac{\varepsilon \mathrm{V}^{2} \mathrm{~b}}{2 \sin ^{2}(\theta)}\left[\frac{\mathrm{D}}{\mathrm{D}-\frac{\mathrm{a}_{2}}{2} \sin (\theta)}-\frac{\mathrm{D}}{\mathrm{D}-\frac{\mathrm{a}_{1}}{2} \sin (\theta)}+\ln \left[\frac{\mathrm{D}-\frac{\mathrm{a}_{2}}{2} \sin (\theta)}{\mathrm{D}-\frac{\mathrm{a}_{1}}{2} \sin (\theta)}\right]\right] \delta \theta$

and

$$
\delta \mathrm{W}_{\mathrm{vdW}}=\frac{\overline{\mathrm{A}} \mathrm{b}}{6 \pi \sin ^{2}(\theta)}\left[\frac{-1+\frac{2 \mathrm{a}}{\mathrm{D}} \sin (\theta)}{2\left(1-\frac{\mathrm{a}}{\mathrm{D}} \sin (\theta)\right)^{2}}+\frac{1}{2}\right] \delta \theta
$$

By substituting equations (3), (4) and (5) into equation (2) the governing equation of main plate rotation calculated as

$\frac{2 \mu \theta}{\mathrm{L}}\left(\mathrm{J}+\mathrm{J}_{\mathrm{c}}\right)-\frac{\varepsilon \mathrm{V}^{2} \mathrm{~b}}{2 \sin ^{2}(\theta)}\left[\frac{\mathrm{D}}{\mathrm{D}-\frac{\mathrm{a}_{2}}{2} \sin (\theta)}-\frac{\mathrm{D}}{\mathrm{D}-\frac{\mathrm{a}_{1}}{2} \sin (\theta)}+\ln \left[\frac{\mathrm{D}-\frac{\mathrm{a}_{2}}{2} \sin (\theta)}{\mathrm{D}-\frac{\mathrm{a}_{1}}{2} \sin (\theta)}\right]-\frac{\overline{\mathrm{A}} \mathrm{b}}{6 \pi \sin ^{2}(\theta)}\left[\frac{-1+\frac{2 \mathrm{a}}{\mathrm{D}} \sin (\theta)}{2\left(1-\frac{\mathrm{a}}{\mathrm{D}} \sin (\theta)\right)^{2}}+\frac{1}{2}\right]=0\right.$

By assuming small rotation which implies $\sin (\theta) \approx \theta$ , equation (6) can be simplified and rewritten to the following dimensionless form:

$\lambda^{2}=\left[\Theta^{3}\left(1+\frac{J_{c}}{J}\right)-\eta \frac{\Theta^{2}}{(1-\Theta)^{2}}\right]\left[\frac{1}{1-\beta \Theta}-\frac{1}{1-\alpha \Theta}+\ln \left(\frac{1-\beta \Theta}{1-\alpha \Theta}\right)\right]^{-1}$

where

$$
\begin{array}{lll}
\eta=\frac{\overline{\mathrm{A}} \mathrm{bL}}{24 \pi \mathrm{D} \theta_{\max }^{3} \mu \mathrm{J}} & (7-\mathrm{a}) & \alpha=\mathrm{a}_{1} / \mathrm{a} \\
\lambda^{2}=\frac{\varepsilon b V^{2} \mathrm{~L}}{4 \theta_{\max }^{3} \mu \mathrm{J}} & (7-\mathrm{b}) & \beta=\mathrm{a}_{2} / \mathrm{a} \\
\theta_{\max }=\mathrm{D} / \mathrm{a} & (7-\mathrm{c}) & \Theta=\theta / \theta_{\max }
\end{array}
$$

Equation (7) can be used to determine the equilibrium angle of the main plate as a function of the applied voltage. The critical values of tilting angle and applied voltage are known as pull-in angle $\left(\Theta_{\mathrm{PI}}\right)$ and pull-in voltage $\left(\mathrm{V}_{\mathrm{PI}}\right)$ respectively. When the applied voltage exceeds $\mathrm{V}_{\mathrm{PI}}$, the electrostatic torque overcomes the mechanical torsion resistance and the main plate will abruptly rotate and pulls into the fixed ground. For the actuator with given geometry, the size-dependent pull-in angle $\left(\Theta_{\mathrm{PI}}\right)$ can be derived from equation (7) by imposing $\frac{\mathrm{d} \lambda^{2}}{\mathrm{~d} \Theta}=0$.

\section{Result and discussion}

It is well-established that the elastic response of a torsional beam depends on the geometry of its crosssection. Hence, two systems with different torsional beam cross-sections e.g rectangular and circular cross-sections are discussed as the case studies. These geometries are of the most common cases that are produced by the industrial fabrication methods. A circular geometry with radius $t$ and a square geometry with half-width $t$ are considered. For the case studies, typical torsional mirrors with $\mathrm{D}=100 \mathrm{~nm}, \mathrm{~L}=200 \mathrm{~nm}$, 
$\mathrm{a}=\mathrm{b}=2000 \mathrm{~nm}$ that operate in $\mathrm{vdW}$ force regimes are investigated. The value of material elastic constant and Hamaker constant are selected as $2.2 \times 10^{-19} \mathrm{~J}$ and $\mu=66 \mathrm{GPa}$, respectively.

In order to investigate the response of nano-actuator, the relation between the tilt angle $(\Theta)$ and applied voltage $(\square)$ has been demonstrated in Fig. 2 neglecting the size effect. As seen, with increasing the voltage, the rotation angle of the nano-actuator is increased. At a critical voltage value, the tilt angle increase even without increasing in applied voltage $\square$ Hence, the pull-in instability occurs and the mainplate abruptly twists to the substrate. Furthermore, this figure reveals that $\mathrm{vdW}$ attraction $(\square)$ reduces the pull-in angle ( $\square$ PI and pull-in voltage ( $\square$ PI $)$ of the system. Interestingly, the intersections of the curves with the vertical axis reveals that vdW attraction produces an initial mirror tilt angle even no voltage difference applied $(\mathrm{V}=0)$.



Figure 2. Relationship of applied voltage and the mirror tilt angle $(\Theta)$. Pull-in occurs for $\mathrm{V}$ values above the critical value of voltage $\left(\lambda_{P I}\right)(\alpha=0.06 \&$ $\beta=0.84$ )

\subsection{Size dependent pull-in angle}

Figure 3 depicts the variation of pull-in angle $\left(\Theta_{\mathrm{PI}}\right)$ of typical mirror $(\alpha=0.06 \& \beta=0.84)$ as a function of the size effect $(l / t)$ for square cross-section mirrors. Similarly, Figure 4 shows the variation of $\Theta_{\mathrm{PI}}$ for circular cross-section systems. These figures reveal that size dependency of material characteristics have a stiffening effect hence increase the pull-in angle of the mirrors. Moreover, it is observed that increasing the beam thickness $(t)$ or decreasing the initial separation $(D)$ reduces the influence of size effect on $\Theta_{\mathrm{PI}}$ of the system. As seen when the thickness of the torsional beam is comparable with the intrinsic material length scale, the size effect might substantially increase the pull-in angle of the system.

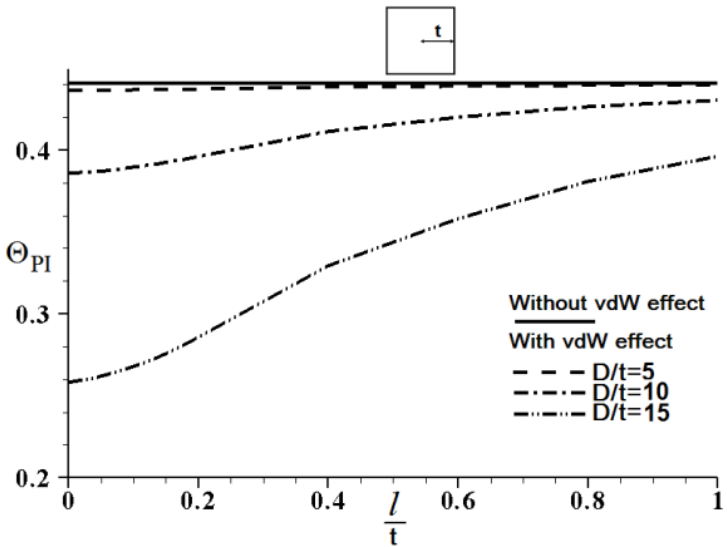

Figure 3. Variation of pull-in angle as function of size effect $(l / t)$ for square cross-section beam $(\alpha=0.06$ $\& \beta=0.84$ )

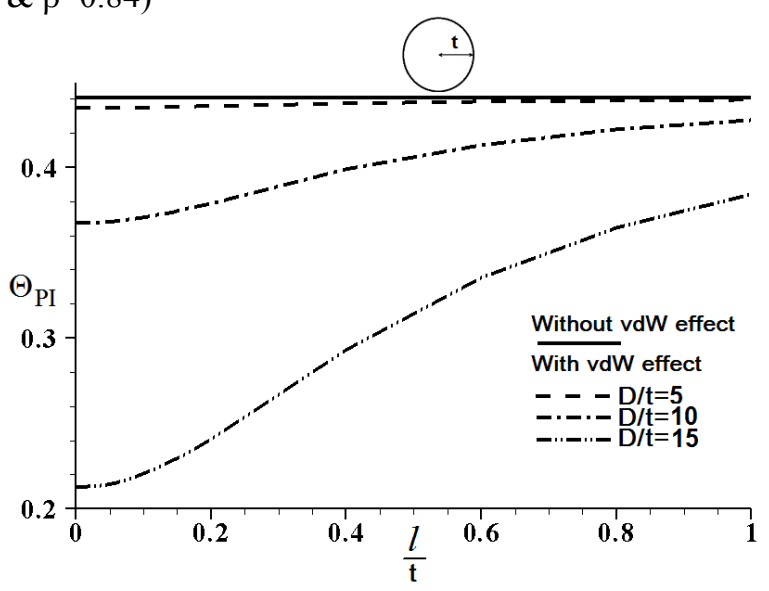

Figure 4. Variation of pull-in angle as function of size effect $(l / t)$ for circular cross-section beam $(\alpha=0.06 \& \beta=0.84)$

\subsection{Size dependent pull-in voltage}

Influence of material length scale on the pull-in voltage of the typical actuator $(\alpha=0.06 \& \beta=0.84)$ is demonstrated in Figures 5 and 6 for square crosssection and circular cross-section systems, respectively. In these figures variation of $\square_{\mathrm{PI}}$ is plotted as a function of $l / t$ for various $D / t$ values. As seen from these figures, size effect increases the instability voltage of the torsional systems. In other words, the pull-in voltage, provided by the modified couple stress model, is higher than that predicted by the classical torsion model. Furthermore, this stiffening effect is more pronounced for larger values of $D / t \square \square$ According to these figures, when the thickness of the torsional beam is comparable with the intrinsic material length scale, classic continuum 
theory is not reliable to compute the pull-in voltage of ultra-small systems made of size-dependent materials.

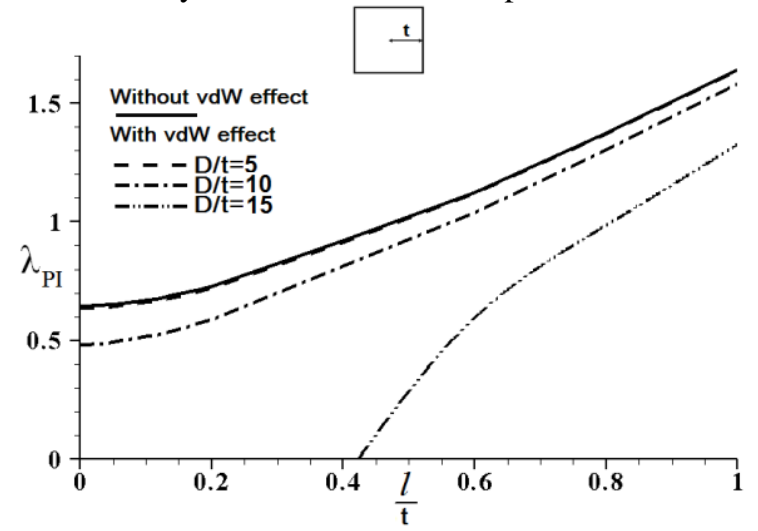

Figure 5. Variation of pull-in voltage as function of size effect $(l / t)$ for square cross-section beam $(\alpha=0.06$ $\& \beta=0.84$

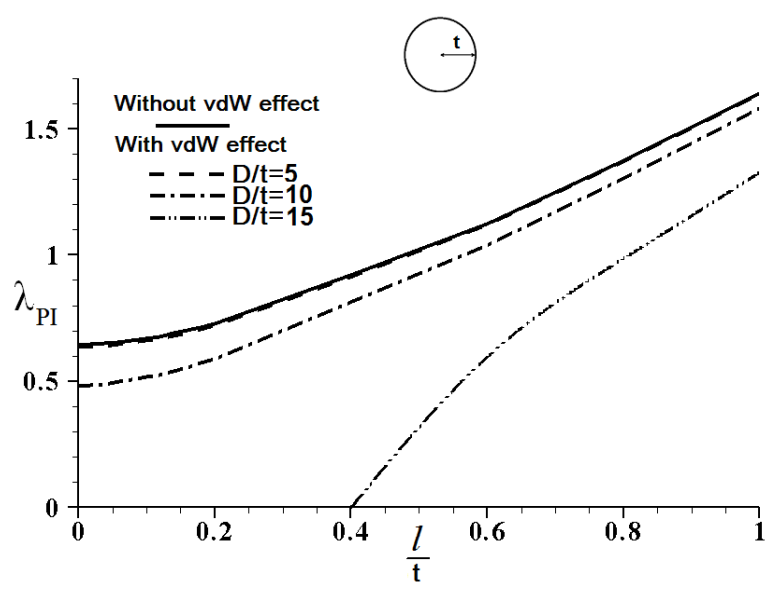

Figure 6. Variation of pull-in voltage as function of size effect $(l / t)$ for circular cross-section beam $(\alpha=0.06 \& \beta=0.84)$

\section{Conclusion}

Herein, the influence of size effect and vdW force on the pull-in performance of the rotational nanoactuators has been investigated using modified couple stress theory. It is found that when the thickness of the torsional beam is comparable with the intrinsic material length scale, the size effect might substantially increase the pull-in angle and pull-in voltage of the torsional actuator.

The pull-in voltage, provided by the modified couple stress theory is higher than that predicted by the classical torsion model. In the absence of the vdW force, the pull-in angle of the model has not been changed by the size effect. However, the pull-in voltage of the mirror is considerably affected by the size effect even if vdW force is omitted from the model. Furthermore,. The critical values of the vdW attraction that can induce stiction in freestanding nano-actuators and corresponding minimum gap have been determined. The size effect decreases the minimum gap due to its stiffening effect on elastic resistance of nano-beams. The developed model is able to predict the experimental results more accurately than the previous classic models. The gap between the experiment and theory can be reduced by using the presented size-dependent model.

\section{References}

[1] H. Toshiyoshi and H. Fujita, Electrostatic microtorsion mirrors for an optical switch matrix, J. Microelectromech. Syst. 1996, 5: 231-237.

[2] J.E. Ford, V.A. Aksyuk, D.J. Bishop, J.A. Walker, Wavelength add-drop switching using tilting micromirrors, J. Lightwave Technol. 1999, 17: 904911.

[3] Y. Nemirovsky and O. Bochobza-Degani, A Methodology and Model for the Pull-In Parameters of Electrostatic Actuators, J. Microelectromech. Syst. 2001, 10(4): 601-615.

[4]J. Cheng, J. Zhe, X. Wu, Analytical and finite element model pull-in study of rigid and deformable electrostatic microactuators, J. Micromech. Microeng. 2004, 14: 57-68.

[5] K.S. Nagapriya, O. Goldbart, I. Kaplan-Ashiri, G. Seifert, R. Tenne, E. Joselevich, Torsional Stick-Slip Behavior inWS2 Nanotubes, Phys. Rev. Lett. 2008, 101: 195501.

[6] J.P. Zhao, H.L. Chen, J.M. Huang and A.Q.Liu, A study of dynamic characteristics and simulations of MEMS torsional micromirror, Sens. Actuat. A 2005, 120: 199-210.

[7] H. Moeenfard and M.T. Ahmadian, Analytical modeling of bending effect on the torsional response of electrostatically actuated micromirrors, Optik 2012, in press.

[8] Z.X. Xiao, X.T. Wu, W.Y. Peng and K.R. Farmer, An angle-based design approach for rectangular electrostatic torsion actuators, J. Microelectromech. Syst. 2001, 10: 561-568

[9] O. Degani and Y. Nemirovsky, Design considerations of rectangular electrostatic torsion actuators based on new analytical pull-in expressions, J. Microelectromech. Syst. 2002, 11: 20-26.

[10] O. Degani, E. Socher, A. Lipson, T. Leitner, D.J. Setter, S. Kaldor and Y. Nemirovsky, Pull-in study of an electrostatic torsion microactuator, J. Microelectromech. Syst. 1998, 7(4): 373-379. 
[11] H. Moeenfard, A. Darvishian, H. Zohoor and M.T. Ahmadian, Characterization of the static behavior of micromirrors under the effect of capillary force, an analytical approach, Proc. Inst. Mech. Eng. Part C J. Mech. Eng. Sci. (2012) doi: 10.1177/0954406211433112.

[12] X.M. Zhang, F.S. Chau, C. Quan, Y.L. Lam and A.Q. Liu, A study of the static characteristics of a torsional micromirror, Sens. Actuators, A 2001, 90: 73-81.

[13] .B. Degani and Y. Nemirovisky, Modeling the pull-in parameters of electrostatic actuators with a novel lumped two degrees of freedom pull-in model, Sens. Actuators, A 2001, 97-98: 569-578.

[14] J. M. Huang, A. Q. Liu, Z. L. Deng, Q. X. Zhang, J. Ahn, A. Asundi, An approach to the coupling effect between torsion and bending for electrostatic torsional micromirrors, Sens. Actuat. A 2004, 115: 159-167.

[15] M.F. Daqaq, E.M. Abdel-Rahman and A.H. Nayfeh, Towards a stable low-voltage torsional microscanner, Microsyst. Technol. 2008,14:725-737.

[16] G. Rezazadeh, F. Khatami, A. Tahmasebi, Investigation of the torsion and bending effects on static stability of electrostatic torsional micromirrors, Microsyst. Technol. 2007, 13: 715-722.

[17] Y. Tadi Beni, A. Koochi, A.S. Kazemi and M. Abadyan, Modeling the Influence of Surface Effect and Molecular Force on Pull-in Voltage of Rotational Nano/Micro Mirror Using 2-DOF Model, Can. J. of Phys. 2012, 90: 963-974.

[18] Y. Tadi Beni, Use of Augmented Continuum Theory for Modeling the Size Dependent Material Behavior of Nano-Actuators, IJST, Transaction of Mechanical Engineering 2012, 36(M1): 41-52.

[19] F. Khatami, Gh. Rezazadeh, Dynamic response of a torsional micromirror to electrostatic force and mechanical shock, Microsyst. Tech. 2009, 15: 535545.

[20] A. Koochi, A.R. Noghrehabadi and M. Abadyan, approximating the effect of van der force on the instability of electrostatic nano-cantilivers, Int. J. Mod. Phys. B 2011, 25: 3965-3976.

[21] R.C. Batra, M. Porfiri and D. Spinello, Effects of van der Waals Force and Thermal Stresses on Pull-in Instability of Clamped Rectangular Microplates Sensors 2008, 8: 1048-1069.

[22] J.M. Dequesnes, S.V. Rotkin and N.R. Aluru, Calculation of pull-in voltages for carbon nanotubebased nanoelectromechanical switches, Nanotechnology 2002, 13: 120.

[23] M. Moghimi Zand, M.T. Ahmadian, B. Rashidian, Dynamic pull-in instability of electrostatically actuated beams incorporating
Casimir and van derWaals forces, Proc. Inst. Mech. Eng. Part C, J. Mech. Eng. 2010, 224 (9): 2037.

[24] W.H. Lin, Y.P. Zhao, Stability and bifurcation behavior of electrostatic torsional NEMS varactor influenced by dispersion forces J. Phys. D: Appl. Phys. 2007, 40: 1649-1654.

[26] J.G. Guo and Y.P. Zhao, Influence of van der Waals and Casimir forces on electrostatic torsional actuators , J. Microelectromech. Sys. 2004, 13(6): 1027-1035.

[27] W.H. Lin and Y.P. Zhao, Dynamics behavior of nanoscale electrostatic actuators, Chin. Phys. Lett. 2003, 20: 2070-2073.

[28] J.G. Guo and Y.P. Zhao, Dynamic stability of electrostatic torsional actuators with van der Waals effect, Int. J. Solids Struct. 2006,43: 675-685.

[29] N.A. Fleck, G.M Muller, M.F. Ashby and J.W. Hutchinson, Strain gradient plasticity: Theory and experiment, Acta Metall. Mater. 1994,42(2):475-487. [30] D.C.C. Lam, F. Yang, A.C.M Chong, J. Wang and P. Tong, Experiments and theory in strain gradient elasticity, J. Mech. Phys. Solids 2003, 51(8):1477-1508.

[31] A.C. Eringen, On differential equations of nonlocal elasticity and solutions of screw dislocation and surface waves, J. Appl. Phys. 1983, 54:47034710.

[32] F. Yang, A.C.M. Chong, D.C.C Lam and P. Tong, Couple stress based strain gradient theory for elasticity, Int. J. Solids Struct. 2002, 39(10): 27312743.

[33] S. Kong, S. Zhou, Z. Nie, K. Wang, Static and dynamic analysis of micro beams based on strain gradient elasticity theory, int. J. Eng. Sci. 2009, 47: 487-498.

[34] S.K. Park and X.L. Gao, Bernoulli-Euler beam model based on a modified couple stress theory $\mathrm{J}$. Micromech. Microeng. 2006, 16: 2355-2359.

[35] H.M. Ma, X.L. Gao and J.N. Reddy, A microstructure-dependent Timoshenko beam model based on a modified couple stress theory, J. Mech. Phys. Solids 2008, 56(12): 3379-3391.

[36] M. Asghari, M.T. Ahmadian, M.H. Kahrobaiyan and M. Rahaeifard, On the size-dependent behavior of functionally graded micro-beams, Mater. Des. 2010, 31: 2324-2329.

[37] L. Wang, Size-Dependent Vibration Characteristics of Fluid-Conveying. Microtubes, J. Fluid. Struct. 2010, 26: 675.

[38] Y. Tadi Beni, A. Koochi, M. Abadyan, Theoretical study of the effect of Casimir force, elastic boundary conditions and size dependency on the pull-in instability of beam-type NEMS, Physica E, 2011, 43: 979-988. 